\title{
A Case of Eosinophilic Chronic Rhinosinusitis Associated with Choroidal Folds and Ocular Motility Disorder
}

\author{
Masahiro Ikeda ${ }^{a} \quad$ Chiharu Iwahashi $^{a} \quad$ Takashi Hirai $^{b} \quad$ Kenji Hattori ${ }^{b}$ \\ Keiichi Mitarai ${ }^{\mathrm{a}}$ \\ aDepartment of Ophthalmology, Sumitomo Hospital, Osaka, Japan; 'bepartment of \\ Otorhinolaryngology - Head and Neck Surgery, Sumitomo Hospital, Osaka, Japan
}

\section{Keywords}

Eosinophilic chronic rhinosinusitis - Choroidal folds - Ocular motility disorder - Anorthopia . Endoscopic sinus surgery

\begin{abstract}
We report a case of eosinophilic chronic rhinosinusitis (ECRS) associated with choroidal folds and ocular motility disorder. A 50-year-old male with rhinosinusitis and bronchial asthma presented with anorthopia of the lower visual field and ocular motility disorder of the left eye. Dilated fundus examination and optical coherence tomography (OCT) revealed wavy choroidal folds in the upper retina. An emergent computed tomography (CT) showed sinusitis, a partial defect of the superior wall of the orbit on the left side, and deformation of the left eye. Based on the clinical findings, the patient was diagnosed with sinusitis complicated by ocular motility disorder. Endoscopic sinus surgery (ESS) was performed. A histopathological examination of the excised polyps showed extensive eosinophil invasion. According to the clinical findings of the nasal polyps, CT images, and peripheral blood tests, he was diagnosed as ECRS. One month after ESS, both ocular movement and anorthopia were improved. The choroidal folds observed using OCT disappeared 2 months after ESS. Although ECRS is rarely associated with ocular complications, bone involvement in sinusitis may result in deformation of the eyeball leading to choroidal folds and ocular motility disorder.




\section{Introduction}

Eosinophilic chronic rhinosinusitis (ECRS) is a subtype of chronic rhinosinusitis, presenting with high-level eosinophil infiltration of nasal polyps. Of note, ECRS is commonly reported with asthma [1]. Ocular complications of ECRS are rarely described; only 2 cases of ECRS associated with optic neuropathy have been reported $[2,3]$. The visual prognosis of these 2 cases was very poor. Following intensive treatment, one patient improved slightly from light perception to finger counting, whereas the other remained stable (no light perception).

In this article, we report a case of ECRS with choroidal folds and ocular motility disorder, which improved after endoscopic sinus surgery (ESS).

\section{Case Report}

A 50-year-old male visited our clinic with anorthopia of the lower visual field and ocular motility disorder of the left eye, which had occurred 3 months before. The patient had been receiving treatment for chronic rhinosinusitis and bronchial asthma. On initial presentation, his best-corrected decimal visual acuity was 1.5 in the right eye and 1.2 in the left eye. Critical flicker fusion frequency was 40 and $34 \mathrm{~Hz}$ in the right and left eye, respectively (normal values in the general population: mean $\pm \mathrm{SD}, 40.0 \pm 1.5 \mathrm{~Hz}$ ). No afferent pupillary defect was observed. A dilated fundus examination of the left eye showed wavy choroidal folds in the upper retina (Fig. 1a). Marked choroidal folds were detected using a vertical scan of optical coherence tomography (OCT) (Fig. 1b). Optic disc edema or retinal atrophy were not observed. Eye movement examination of the left eye showed restricted elevation of the globe.

Based on the clinical findings, we suspected a space-occupying retrobulbar mass and performed orbital computed tomography (CT). The CT scan showed pansinusitis, a partial defect of the left superior wall of the orbit, and deformation of the left eye (Fig. 2a). Thus, we considered that the deformation was due to extensive rhinosinusitis, causing visual disturbance, including the wavy choroidal folds in the upper retina and ocular motility disorder.

We consulted an otorhinolaryngologist, and ESS was performed 1 week later. Intraoperatively, the ethmoid, maxillary, frontal, and sphenoid sinuses were filled with polyps and highly viscous mucosal fluid. Especially, the left frontal sinus was almost completely filled with polyps. A histopathological examination of the excised polyps showed extensive eosinophil invasion (Fig. 2b). Necrotizing granulomatosis was not observed. The laboratory data showed an elevation of IgE (19.8\%) prior to the treatment of bronchial asthma. The patient was diagnosed as ECRS.

One month after ESS, both ocular movement and anorthopia had improved rapidly. The choroidal folds observed using OCT disappeared 2 months after ESS. However, the marks of the previous parallel horizontal retinal and choroidal folds on the fundus images persisted for 1 year (Fig. 3a, b). ECRS was treated with antihistamine, antileukotriene, and rhinenchysis for 2 years after ESS.

\section{Discussion}

Choroidal folds are undulations or wrinkles in the retinal pigment epithelium, Bruch's membrane, and inner choroid. They have been observed in eyes with orbital disease, choroidal tumors, ocular hypotony, scleritis, acute Vogt-Koyanagi-Harada disease, and 
papilledema [4-6]. Factors that cause the normal expansion of Bruch's membrane to shorten are considered to contribute to the development of choroidal folds. In cases with scleritis, acute Vogt-Koyanagi-Harada disease, and papilledema, increased choroidal thickness may be related to choroidal folds. Typically, folds radiating $360^{\circ}$ from the optic disc are observed [6]. On the other hand, in cases with orbital disease, the mass effect of the lesion may be related to the development of choroidal folds. Therefore, the morphology of the folds varies according to the direction of compression force.

In the present case, parallel horizontal retinal and choroidal folds in the upper retina suggested that the globe was pressed from above. Subsequently, we performed an urgent orbital CT and diagnosed the patient as sinusitis. Orbital infection associated with sinusitis is liable to loss of vision [7]. Although this case had not decreased visual acuity, we consulted otorhinolaryngologists to exclude an inflammatory cause exacerbated by bacterial or fungal infection. Because the patient's visual acuity was not affected and no afferent pupillary defect was observed, we considered that anorthopia of the lower visual field and ocular motility disorder were related to the space-occupying retrobulbar mass effect, rather than the compressive or infectious optic neuropathy. Therefore, ESS was scheduled 1 week later.

A histopathological examination of the excised polyps revealed extensive eosinophil invasion. The diagnosis of severe ECRS was finally reached based on the clinical findings of the nasal polyps, CT images, peripheral blood tests, and comorbid bronchial asthma [8]. Patients with severe ECRS have a high risk of frequent recurrence. In this case, ECRS was controlled pharmacologically for 2 years after ESS; however, careful follow-up is required.

Ocular complications of ECRS have not been thoroughly investigated. Only 2 case reports have been described. Garg et al. [2] reported a case of visual loss secondary to ECRS treated with steroids and radical sphenoethmoidal clearance. The visual prognosis in this case was very poor, and only slight improvement was observed from light perception to finger counting. Kurimoto et al. [3] reported another case of ECRS associated with optic neuropathy which remained stable (no light perception) in spite of emergency ESS and steroid therapy. The pathogenesis of these cases remains unclear. Considering the persistence of visual impairment after optic nerve decompression, a possible mechanism may include optic neuritis secondary to adjacent inflammatory sinus disease. In the present case, visual acuity was not affected, suggesting that mechanical compression of the globe may simply cause visual disturbance.

After ESS, rapid improvement of the ocular motility was observed. Prior to the operation, the space-occupying retrobulbar mass prevented the patient from looking upward. Improvement in the choroidal folds was also noted 2 months later using OCT. However, the marks of the choroidal folds on the fundus images persisted for 1 year. This finding reflects the stiffness of Bruch's membrane, containing considerable elastin and collagen [9]. Choroidal folds were observed in the acute stage of Vogt-Koyanagi-Harada disease and disappeared promptly on fundus images and OCT $[10,11]$. In our case, choroidal folds gradually occurred; therefore, improvement on fundus images may be delayed.

This is the first case report of choroidal folds and ocular motility disorder associated with ECRS. Our case demonstrated rapid improvement in ocular motility and choroidal folds after ESS.

\section{Conclusion}

We reported a patient with ECRS who presented with choroidal folds and ocular motility disorder. Although ECRS is rarely associated with ocular complications, bone involvement in 
sinusitis may result in deformation of the eyeball leading to choroidal folds and ocular motility disorder. ESS was very effective in improving ocular motility and choroidal folds.

\section{Acknowledgments}

We thank Dr. Fujita for performing the histological analysis of the samples obtained from the patient's sinuses.

\section{Statement of Ethics}

The authors have no ethical conflicts to disclose.

\section{Disclosure Statement}

The authors report no conflicts of interest in this work.

\section{Funding Sources}

There were no funding sources.

\section{Author Contributions}

M.I. and C.I. were the major contributors to the manuscript. T.H. was part of the otorhinolaryngologists who were in charge of the patient's care. K.H. was the nose surgeon who performed the surgical intervention on the patient and advised on the writing of the manuscript. K.M. was the consultant ophthalmologist and advised on the writing of the manuscript. All authors read and approved the final manuscript.

\section{References}

1 Haruna S, Nakanishi M, Otori N, Moriyama H. Histopathological features of nasal polyps with asthma association: an immunohistochemical study. Am J Rhinol. 2004 May-Jun;18(3):165-72.

2 Garg A, Das-Bhaumik R, Nesbitt AD, Levene AP, Joshi N, Grant WE, et al. Visual loss secondary to eosinophilic mucin rhinosinusitis in a woman: a case report. J Med Case Reports. 2010 Oct;4(1):350.

3 Kurimoto T, Tonari M, Ishizaki N, Matsuo J, Oku H, Sugasawa J, et al. A case of eosinophilic chronic rhinosinusitis associated with optic neuropathy. Clin Ophthalmol. 2011;5:853-6.

4 Newell FW. Choroidal folds. The seventh Harry Searls Gradle Memorial lecture. Am J Ophthalmol. 1973 Jun;75(6):930-42.

5 Friberg TR. The etiology of choroidal folds. A biomechanical explanation. Graefes Arch Clin Exp Ophthalmol. 1989;227(5):459-64.

6 Tsuboi K, Nakai K, Iwahashi C, Gomi F, Ikuno Y, Nishida K. Analysis of choroidal folds in acute VogtKoyanagi-Harada disease using high-penetration optical coherence tomography. Graefes Arch Clin Exp Ophthalmol. 2015 Jun;253(6):959-64.

7 Gupta AK, Bansal S, Gupta A, Mathur N. Visual loss in the setting of allergic fungal sinusitis: pathophysiology and outcome. J Laryngol Otol. 2007 Nov;121(11):1055-9. 
Ikeda et al.: A Case of Eosinophilic Chronic Rhinosinusitis Associated with Choroidal Folds and Ocular Motility Disorder

8 Tokunaga T, Sakashita M, Haruna T, Asaka D, Takeno S, Ikeda H, et al. Novel scoring system and algorithm for classifying chronic rhinosinusitis: the JESREC Study. Allergy. 2015 Aug;70(8):995-1003.

9 Del Priore LV. Stiffness of retinal and choroidal tissue: a surface wrinkling analysis of epiretinal membranes and choroidal folds. Am J Ophthalmol. 2006 Sep;142(3):435-40.

10 Shinoda K, Imamura Y, Matsumoto CS, Mizota A, Ando Y. Wavy and elevated retinal pigment epithelial line in optical coherence tomographic images of eyes with atypical Vogt-Koyanagi-Harada disease. Graefes Arch Clin Exp Ophthalmol. 2012 Sep;250(9):1399-402.

11 Tanigawa M, Tsukahara Y, Yamanaka H. A case of acute posterior multifocal placoid pigment epitheliopathy demonstrating Vogt-Koyanagi-Harada disease-like optical coherence tomography findings in the acute stage. Case Rep Ophthalmol. 2013 Oct;4(3):172-9.
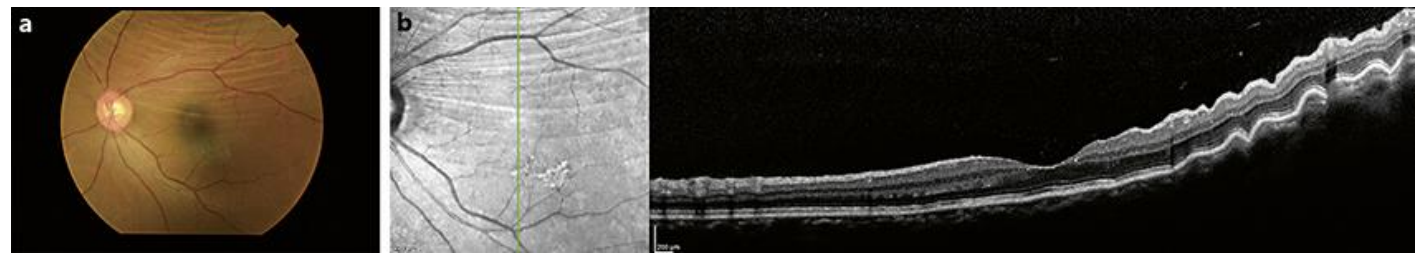

Fig. 1. a Images at initial presentation. Fundus color photography showing parallel horizontal retinal and choroidal folds in the upper retina. b Markedly folded Bruch's membrane, retinal pigment epithelium, and choroid are noted using infrared imaging and a vertical scan of optical coherence tomography.
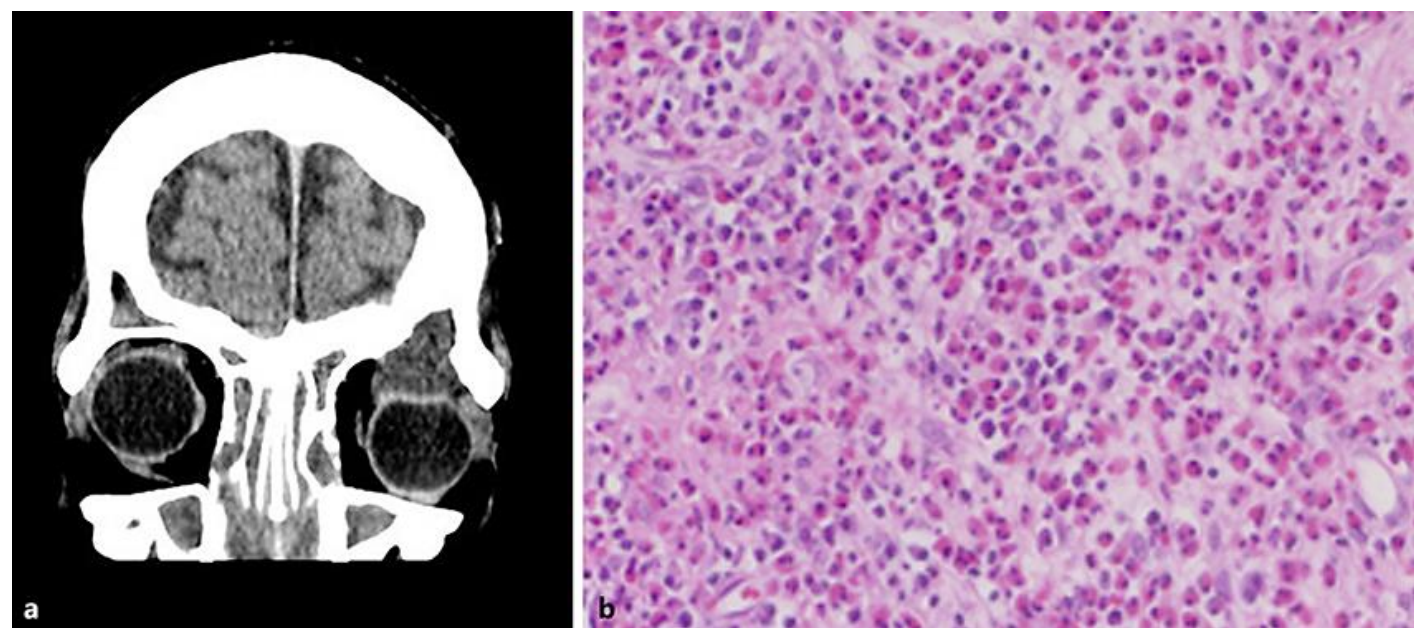

Fig. 2. a Computed tomography image of the orbits revealed deformation of the left eye due to an orbital mass. Sinusitis and a partial defect of the superior wall of the orbit on the left side were also detected. b Photomicrograph of sections obtained from the nasal polyp showing extensive eosinophil invasion. HE. $\times 400$. 
Case Reports in
Ophthalmology

Case Rep Ophthalmol 2019;10:89-94

DOI: $10.1159 / 000497270$

C) 2019 The Author(s). Published by S. Karger AG, Basel www.karger.com/cop

Ikeda et al.: A Case of Eosinophilic Chronic Rhinosinusitis Associated with Choroidal Folds and Ocular Motility Disorder
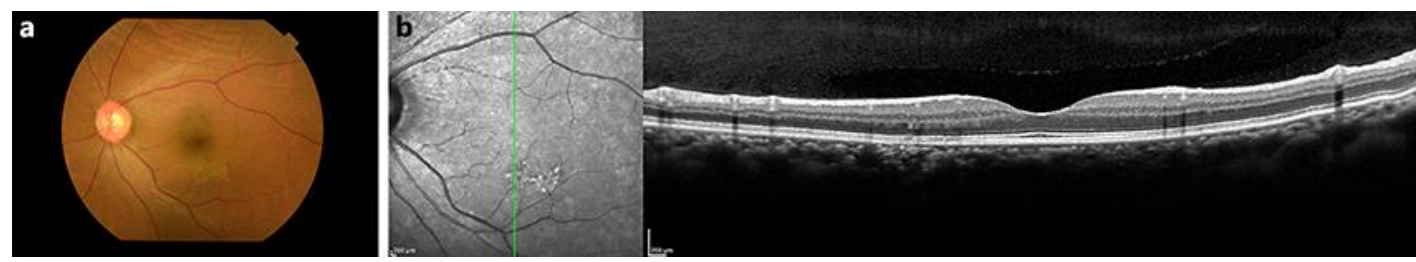

Fig. 3. a Post-treatment images of the chorioretinal structure. The marks of previous parallel horizontal retinal and choroidal folds are observed. $\mathbf{b}$ Infrared imaging and a vertical scan of optical coherence tomography showing completely resolved choroidal folds. 